\title{
Swift heavy ion induced nano-dimensional phase separation in liquid immiscible binary $\mathrm{Mn}-\mathrm{Bi}$
}

\author{
S.K. Srivastava ${ }^{\mathrm{a}, *}$, S.A. Khan $^{\mathrm{b}}$, P. Sudheer Babu ${ }^{\mathrm{c}}$, D.K. Avasthi ${ }^{\mathrm{b}}$ \\ ${ }^{a}$ Department of Physics and Meteorology, Indian Institute of Technology, Kharagpur 721302, India \\ ${ }^{\mathrm{b}}$ Inter University Accelerator Centre, Aruna Asaf Ali Marg, P.O. Box 10502, New Delhi 110067, India \\ ${ }^{\mathrm{c}}$ Department of Physics, RGUKT, Nuzvid 521201, India
}

\section{A R T I C L E I N F O}

\section{Article history:}

Available online 13 March 2014

\section{Keywords:}

Swift heavy ion

Liquid immiscibility

Phase separation

Dispersed nanostructures

\begin{abstract}
A B S T R A C T
Pulsed laser deposited $60 \mathrm{~nm}$ thin film of homogeneous $\mathrm{Mn}_{0.82} \mathrm{Bi}_{0.18}$ composite has been irradiated by $100 \mathrm{MeV}$ Au ions at fluence $1 \times 10^{13}$ ions $/ \mathrm{cm}^{2}$, and investigated by field emission scanning electron microscopy, X-ray diffraction, magnetic hysteresis, X-ray photoelectron spectroscopy, and nanoindentation measurements. Dispersed nanostructures of soft Bi-rich phase of about $20 \mathrm{~nm}$ diameter emerged in a hard Mn-rich matrix on irradiation. Such structures, as synthesized by the present novel swift heavy ion irradiation approach, are usable as self-lubricating thin films.
\end{abstract}

(c) 2014 Elsevier B.V. All rights reserved.

\section{Introduction}

Materials composed of dispersed soft metal nanoparticles in hard metal matrix have potential applications as solid lubricants in advanced bearing systems [1,2]. Attempts are made to synthesize such structures by rapid thermal annealing of liquid immiscible binaries at earth $[3,4]$ and in space $[5,6]$, but the density difference induced stratification and Marangoni motion lead to stratification and coarse phase separation. In this context, a different materials synthesis technique, viz., ion beam processing, is worth viewing upon. Swift heavy ions (SHI's), which have immense potential of liquefying metals in nanosized latent tracks and cool them extremely rapidly at a rate of about $10^{14} \mathrm{~K} / \mathrm{s}[7,8]$, could be a very suitable means to synthesize such phase-separated nanostructures. Precipitation of $\mathrm{C}$ nanoclusters in ion-irradiated polymers and gels [9,10], Fe and Co nanoparticles in polymers [11], and noble metal nanoparticles embedded in oxide matrices [12] are some reports of nanophase formation using ion beams. As far as studies on liquid immiscible elements are concerned, there are reports only on the ion irradiation of multilayers: (i) by Enrique et al. [13], who report a semi-coherent decomposition of nanostructures on $1 \mathrm{MeV} \mathrm{Kr}, 125^{\circ} \mathrm{C}$ irradiation of $\mathrm{Ag} / \mathrm{Cu}$ multilayer, and (ii) by Gao et al. [14], who observed an irradiation resistance and immiscibility in $\mathrm{W} / \mathrm{Cu}$ multilayers.

The Mn-Bi phase diagram possesses a liquid miscibility gap in 55-100 atomic percent $\mathrm{Mn}$, and $1235-1800{ }^{\circ} \mathrm{C}$ temperature,

\footnotetext{
* Corresponding author. Tel.: +91 3222 283854, mobile: +91 9735444091.

E-mail address: sanjeev@phy.iitkgp.ernet.in (S.K. Srivastava).
}

ranges [15]. A homogeneous mixture of $\mathrm{Mn}$ and $\mathrm{Bi}$ with $\mathrm{Mn}$ as the majority component is expectedly highly susceptible to the mechanism described by the thermal spike model [8] because of the high e-p coupling - about $10^{3} \mathrm{~W} \mathrm{~cm}^{-3} \mathrm{~K}^{-1}$ in $\mathrm{Mn}$ as opposed to $125 \mathrm{~W} \mathrm{~cm}^{-3} \mathrm{~K}^{-1}$ for $\mathrm{Fe}$ [7], for which lattice temperatures are reported in [8]. Thus, SHI irradiation of homogeneous Mn-Bi composite thin film has a potential to generate structures with soft, possibly Bi, nanoparticles dispersed in hard, possibly Mn, matrix.

In the present work, we synthesize a thin-film structure with soft $\mathrm{Bi}$ nanoparticles dispersed in a hard Mn-rich matrix by $100 \mathrm{MeV} \mathrm{Au}$ ion irradiation of a homogeneous $\mathrm{Mn}_{0.82} \mathrm{Bi}_{0.18}$ composite thin film.

\section{Experimental}

Appropriate amounts of $\sim 99.99 \%$ pure metallic Mn and Bi from Alfa Aesar were first melted together in an argon arc furnace and cooled to form a solid composite, which was then crushed and pelletized. A thin film was deposited on a quartz substrate from this pellet by pulsed laser deposition. The composition $\mathrm{Mn}_{0.82} \mathrm{Bi}_{0.18}$ of the film was determined by energy dispersive X-ray spectroscopy. The film was then cut into two parts, one of which was irradiated by $100 \mathrm{MeV} \mathrm{Au}$ ions at $1 \times 10^{13}$ ions $/ \mathrm{cm}^{2}$ fluence using the $15 \mathrm{UD}$ Pelletron accelerator at IUAC, New Delhi. The electronic and nuclear energy losses, $S_{\mathrm{e}}$ and $S_{\mathrm{n}}$, respectively, of the ion in the composite, as calculated using TRIM [16], are $\sim 22.8 \mathrm{keV} / \mathrm{nm}$ and $\sim 0.6 \mathrm{keV} / \mathrm{nm}$, respectively, suggesting the dominance of $S_{e}$ and applicability of the thermal spike model. The samples were characterized by field emission scanning electron microscopy (FESEM), X-ray diffraction 
(XRD), X-ray reflectivity (XRR), magnetization, X-ray photoelectron spectroscopy (XPS) and nanoindentation measurements. FESEM was performed using Carl Zeiss SUPRA 40 Field Emission Scanning Electron Microscope, the XRD, using Philips X'Pert diffractometer with $\mathrm{Cu} \mathrm{K} \alpha$ radiation, the XRR, in the P08 beamline of PETRA III, DESY, Hamburg, Germany, the magnetization versus magnetic field, using Quantam Design SQUID magnetometer, the XPS, using PHI VersaProbeII system, and the nanoindentation using Hysitron TriboIndenter.

\section{Results and discussion}

\subsection{Field emission scanning electron microscopy}

The FESEM images of the pristine and irradiated samples are shown in Fig. 1. The pristine sample displays homogeneity in the surface morphology indicating that $\mathrm{Bi}$ and $\mathrm{Mn}$ are in a mixed state on deposition. After irradiation, the surface morphology features brighter nanostructures of $\sim 20 \mathrm{~nm}$ sizes and presumably of Bi-rich phase dispersed in a matrix of darker, presumably Mn-rich, phase. This observation is an evidence of nanophase separation induced in $\mathrm{Mn}_{0.82} \mathrm{Bi}_{0.18}$ film by the SHI.

\subsection{X-ray diffraction and X-ray reflectivity}

The XRD patterns of the pristine and irradiated samples are shown in Fig. 2. The pattern for the pristine sample is quite featureless, except for one small peak corresponding to Mn (112) [17]. This peak in fact occurs at an angle $0.14^{\circ}$ greater than the pure Mn value, indicating that $\mathrm{Mn}$ is embedded with atoms of $\mathrm{Bi}$, which has smaller lattice constant than that of $\mathrm{Mn}$, an observation in consistence with the FESEM picture. Traces of $\mathrm{Bi}$ (202) and $\mathrm{MnO}_{2}$ (111) peaks can also be identified, but they are very broad and of low intensity. It means $\mathrm{Bi}$ is not in its crystalline form; rather $\mathrm{Bi}$ atoms are homogeneously distributed in Mn matrix. The minor oxidation of Mn is insignificant. On irradiation, the above peaks get enhanced and a new shifted Mn (211) peak emerges, indicating a separation of Mn-rich and Bi phases. The thickness of the film was determined to be $60 \mathrm{~nm}$ by XRR. The inset of Fig. 2 shows the XRR curve and its fit using PARRATT32 code for the pristine sample as a representative data.

\subsection{Magnetic hysteresis}

Fig. 3 shows the magnetic hysteresis curves for pristine and irradiated samples at $10 \mathrm{~K}$ (Fig. 3a) and at $300 \mathrm{~K}$ (Fig. 3b). The linear component of the curve, arising collectively from possible and unresolvable paramagnetism of $\mathrm{Mn}$ and diamagnetism of $\mathrm{Bi}$ and substrate, has been subtracted from the curves. At both the

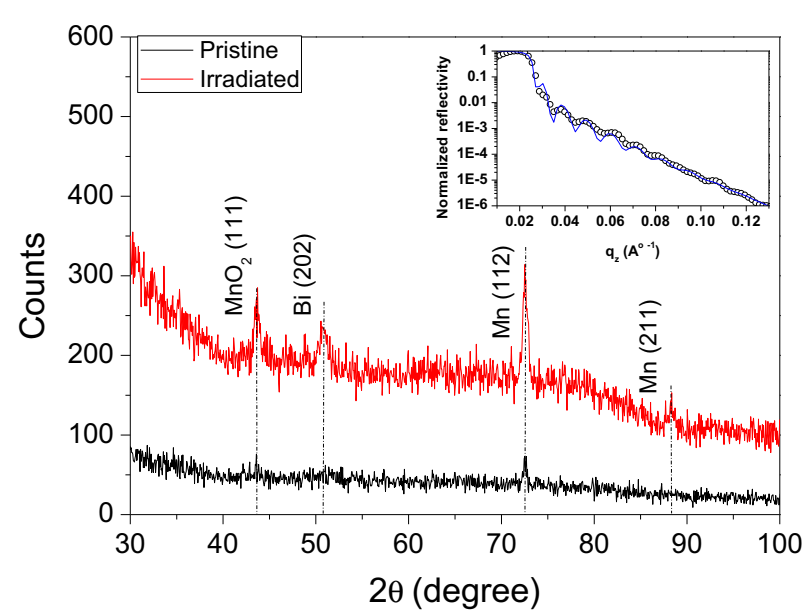

Fig. 2. XRD patterns of pristine and irradiated samples. Inset: XRR curve and its fit for the pristine sample.

temperatures, the pristine sample exhibits a hysteresis loop, the area of the loop being larger at the lower temperature. This temperature dependence of the hysteresis loop area is indicative of presence of ferromagnetism in the pristine sample. Pure $\mathrm{Mn}$ is known to be paramagnetic [18], while $\mathrm{Mn}$ with $\mathrm{Bi}$, as in BiMn phase [19], is ferromagnetic. Thus, it can be inferred that the pristine sample is a composite of $\mathrm{Mn}$ and $\mathrm{Bi}$, as evidenced also by FESEM and XRD. On irradiation, there is a further reduction in the coercivity as well as in the saturation magnetization at both temperatures. This is indicative of a loss of ferromagnetism and appearance of paramagnetism due to separation of Mn from MnBi composite on irradiation, an inference also obtained from the FESEM and XRD results.

\subsection{X-ray photoelectron spectroscopy}

Fig. 4 displays the XPS spectra for pristine and irradiated samples in $\mathrm{Mn} 2 \mathrm{p}, \mathrm{O} 1 \mathrm{~s}$ and $\mathrm{Bi} 4 \mathrm{f}$ regions. The $\mathrm{Bi}_{4 \mathrm{f} / 2}$ peak for the pristine sample occurs at $158.1 \mathrm{eV}$, while the elemental Bi peak occurs at $157.0 \mathrm{eV}$ [20]. This peak must arise due to $\mathrm{Mn}$ neighborhood around $\mathrm{Bi}$ atoms, indicating a mixture of $\mathrm{Bi}$ and $\mathrm{Mn}$. The $\mathrm{Mn} 2 \mathrm{p}_{3}$ 2 peak at $639.9 \mathrm{eV}$ is also correspondingly higher than the pure Mn value [21]. A weak hump at $641.6 \mathrm{eV}$ is due to a small amount of $\mathrm{MnO}_{2}$. The $529.0 \mathrm{eV} \mathrm{O} 1 \mathrm{~s}$ peak corresponds to adsorbed oxygen [22], and the weaker, $531.6 \mathrm{eV}$ peak corresponds to $\mathrm{MnO}_{2}$ [22]. On irradiation, the Bi peak shifts by $-1.1 \mathrm{eV}$ to pure Bi value, indicating the phase separation of Bi. A similar but opposite $(+1.4 \mathrm{eV})$ shift in the main Mn peak after irradiation is in accordance with the Bi peak shift [23]. This peak still not occurring at the pure Mn position $(638.7 \mathrm{eV})$ indicates the presence of $\mathrm{Mn}$-rich $\mathrm{Mn}-\mathrm{Bi}$ phase. The $\mathrm{O}$
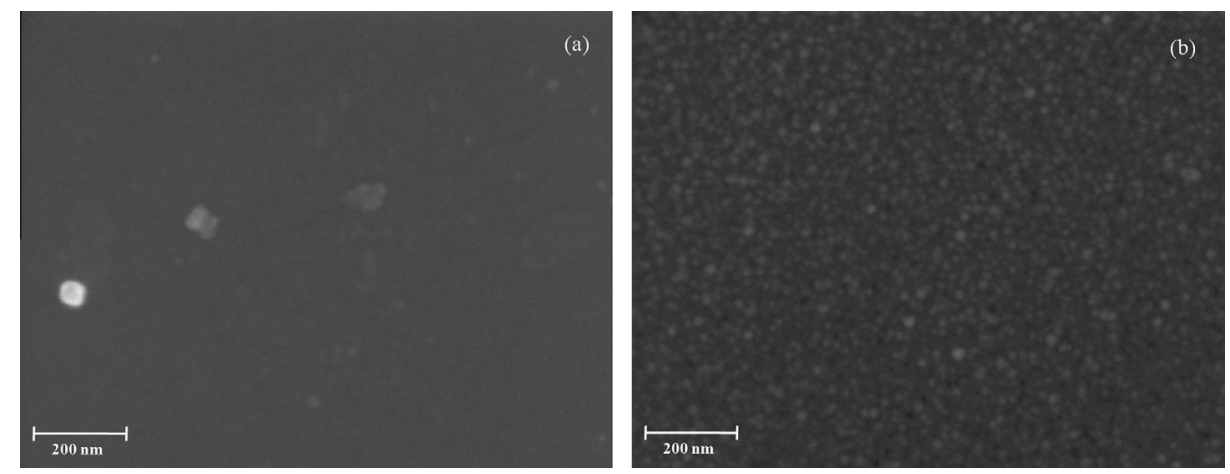

Fig. 1. FESEM images of (a) pristine and (b) irradiated samples. 

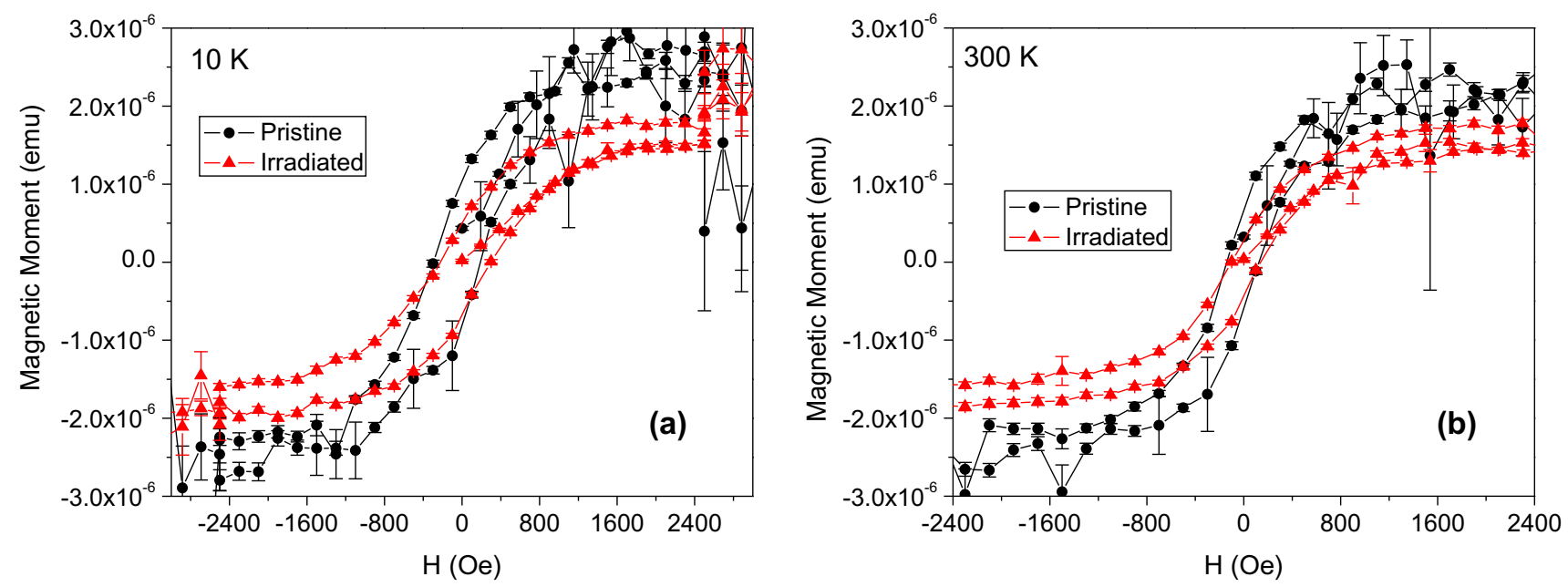

Fig. 3. Magnetic hysteresis (M-H) curves for pristine and irradiated samples at (a) $10 \mathrm{~K}$ and (b) $300 \mathrm{~K}$.

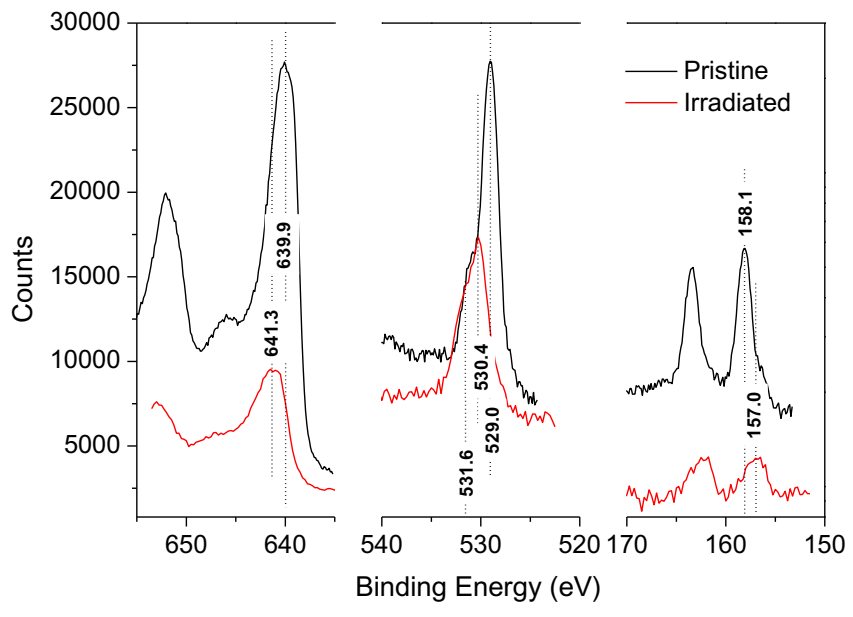

Fig. 4. XPS spectra of pristine and irradiated samples in $\mathrm{Bi} 4 \mathrm{f}_{7 / 2}, \mathrm{O} 1 \mathrm{~s}$ and $\mathrm{Mn} 2 \mathrm{p}_{3 / 2}$ regions of binding energy.

1s peaks, occurring at $530.4 \mathrm{eV}$ and $531.6 \mathrm{eV}$, still correspond to adsorbed oxygen and $\mathrm{MnO}_{2}$, respectively. Thus, the XPS results suggest a phase separation of $\mathrm{Bi}$ and Mn-rich phases on irradiation.

\subsection{Nanoindentation}

The load-displacement curves for pristine and irradiated samples for a number of measurement attempts are shown in Fig. 5. For the pristine sample, almost the same curves are reproduced in all attempts, indicating this to be a laterally homogeneous film. The mean hardness as derived from hardness versus contact depth curve (not shown) is $10.3 \mathrm{GPa}$. From the curves after irradiation, two observations can be made. (i) There are many curves in different attempts, indicating lateral inhomogeneity, akin to a structure with soft phases embedded in a hard matrix. (ii) The unloading curves for irradiated samples have smaller slopes than the pristine ones, indicating a softening in the thin film on irradiation [24]. This is corroborated by the $5.6 \mathrm{GPa}$ mean hardness obtained by the hardness versus contact depth measurement.

\subsection{Possible mechanism}

The results from the various characterizations discussed above lead to a common conjecture that the SHI irradiation has induced

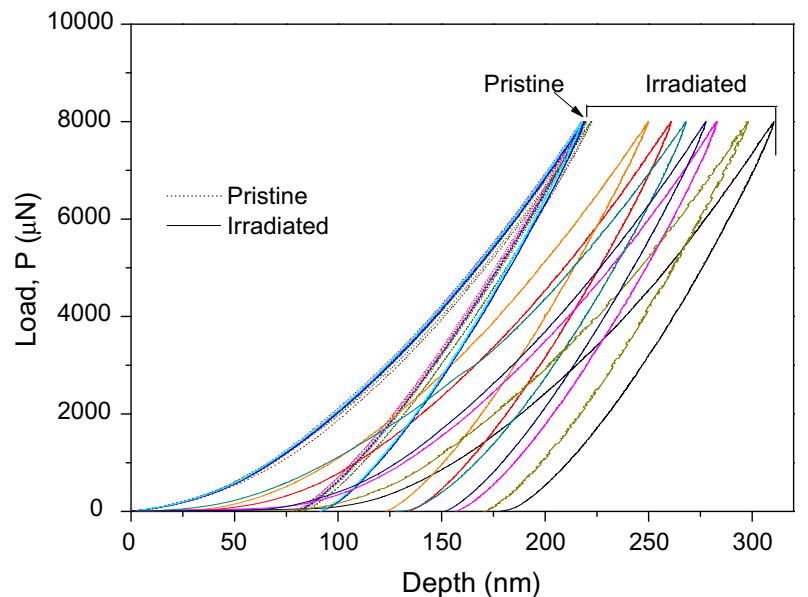

Fig. 5. Load-displacement curves in various attempts for pristine (dotted lines) and irradiated (solid lines) samples.

phase separation of Bi and Mn-rich Mn-Bi phases in a homogeneous $\mathrm{Mn}-\mathrm{Bi}$ composite. Since the $S_{\mathrm{e}}$ is dominant and the system is metallic, the energy transfer mechanism from electronic to lattice subsystem can be assumed to be the e-p coupling [8] as described by the thermal spike model. It is reported earlier [8] that lattice temperatures of $\mathrm{Fe}$ can be raised to about $2000{ }^{\circ} \mathrm{C}$ in about $3 \mathrm{~nm}$ radius cylinder around the $200 \mathrm{MeV} \mathrm{Au}$ ion $\left(S_{\mathrm{e}} \sim 45.8 \mathrm{keV} /\right.$ $\mathrm{nm})$; it takes about $1 \times 10^{13}$ ions $/ \mathrm{cm}^{2}$ to fill the whole sample with ion tracks of this diameter. Since the e-p coupling strength $\left(10^{3}-\right.$ $\mathrm{W} \mathrm{cm}{ }^{-3} \mathrm{~K}^{-1}$ ) in $\mathrm{Mn}-\mathrm{Bi}$ is about an order of magnitude greater than that for Fe (125 W cm $\left.{ }^{-3} \mathrm{~K}^{-1}\right)$, it can be safely assumed (i) that the miscible liquid temperatures ( $>1800 \mathrm{~K}$ ) can be easily achieved by the present $S_{\mathrm{e}}$ value, and (ii) that the $1 \times 10^{13}$ ions $/ \mathrm{cm}^{2}$ fluence is sufficient to fill the whole sample with ion tracks in the present case also. The molten Mn Bi liquid, while passing through the liquid immiscibility region on rapid cooling [15], creates separation of immiscible liquid phases, freezes subsequently, and thus leads to the formation of the observed structure.

\section{Conclusion}

In the present work, pulsed laser deposited homogeneous $\mathrm{Mn}_{0.82} \mathrm{Bi}_{0.18}$ thin film was irradiated by $100 \mathrm{MeV} \mathrm{Au}$ ions at $1 \times 10^{13}$ ions $/ \mathrm{cm}^{2}$ fluence. The FESEM pictures reveal the 
emergence of $\sim 20 \mathrm{~nm}$ diameter nanostructures of Bi-rich phase dispersed in Mn-rich matrix on irradiation. The post-irradiation enhancement in Bi and Mn-rich Mn-Bi XRD peaks and the emergence of a new Mn-rich Mn-Bi peak corroborate the phase separation phenomenon, while XRR of the pristine samples reveals the thickness of the film to be $60 \mathrm{~nm}$. The persistence of magnetic hysteresis loop with reduced area and symmetric Mn and Bi XPS peak shifts also suggest the $\mathrm{Mn}$-rich $\mathrm{Mn}-\mathrm{Bi}$ phase separation. A reduction in the mean hardness and emergence of lateral inhomogeneity is an evidence of formation of softer phases dispersed in a harder matrix on irradiation. Thus, all the characterization measurements collectively suggest that $100 \mathrm{MeV}$ ion irradiation of a homogeneous mixture of $\mathrm{Mn}$ and $\mathrm{Bi}$ has led to the synthesis of Bi nanostructures dispersed in Mn-rich Mn-Bi matrix.

\section{Acknowledgements}

This work was funded by the Department of Science and Technology through the project SR/FTP/PS-59/2-7. The authors would like to acknowledge S. K. Ray, IIT Kharagpur for the PLD deposition and the Pelletron group of IUAC, New Delhi for irradiation. The team members, particularly P. Swain and R. Das, of the DST funded XPS facility in the Department of Physics and Meteorology, IIT Kharagpur are also acknowledged for the XPS measurements.

\section{References}

[1] B. Predel et al., in: H.U. Walter, M.F. Ashhy (Eds.), Decomposition of Alloys: The Early Stages, Springer, Berlin, 1987, p. 517.

[2] L. Ratke, S. Diefenbach, Mater. Sci. Eng. 15 (1995) 263.

[3] Y. Yong, X. Li, Trans. ASME 129 (2007) 252

[4] X. Fang, Z. Fan, Mater. Sci. Technol. 21 (2005) 366.

[5] T. Carlberg, H. Frederiksson, Metall. Trans. A 11A (1980) 1665.

[6] H.C. deGroh III, H.B. Probst, J. Spacecraft Rockets 26 (1989) 476.

[7] Z.G. Wang, Ch. Dufour, E. Paumier, M. Toulemonde, J. Phys. Condens. Matter 6 (1994) 6733.

[8] S.K. Srivastava, D.K. Avasthi, W. Assmann, Z.G. Wang, H. Kucal, E. Jacquet, H.D. Carstanjen, M. Toulemonde, Phys. Rev. B 71 (2005) 193405.

[9] S.K. Srivastava, D.K. Avasthi, E. Pippel, Nanotechnology 17 (2006) 2518.

[10] Amit Kumar et al., Nucl. Instr. Meth. Phys. Res. B 244 (2006) 23.

[11] B.Z. Rameev et al., Microelectronic Eng. 69 (2003) 330.

[12] S.A. Khan, S.K. Srivastava, D.K. Avasthi, J. Phys. D: Appl. Phys. 45 (2012) 375304.

[13] R. Enrique, F. Wu, P. Bellon, Surf. Coat. Technol. 150 (2002) 1.

[14] Y. Gao et al., J. Nucl. Mater. 413 (2011) 11.

[15] T.B. Massalski, Binary Alloy Phase Diagrams, second ed., vol. 1, 1990, A.S.M. International, p. 759.

[16] J.P. Biersack, Nucl. Instr. Meth. Phys. Res. B 27 (1987) 21.

[17] Inorganic Materials Database - AtomWork, <http://crystdb.nims.go.jp/ index_en.html>.

[18] Tables de Constantes et Données Numérique, Volume 7, Relaxation Paramagnetique, Masson, Paris, 1957.

[19] W. Hui et al., Trans. Nonferrous Met. Soc. China 13 (2003) 1405.

[20] Yunsheng Dai et al., Catal. Lett. 123 (2008) 307.

[21] M.C. Besinger et al., Appl. Surf. Sci. 257 (2011) 2717.

[22] <www.xpsdata.com/XI_BE_Lookup_table.pdf>.

[23] J.J. Kolodzeij, T.E. Madey, J.W. Keister, J.E. Rowe, Phys. Rev. B 62 (2000) 5150.

[24] M.F. Doerner, W.D. Nix, J. Mater. Res. 1 (1986) 601. 